\title{
Doppler-broadening measurements of positron annihilation with high-momentum electrons in pure elements
}

\author{
R.S. Brusa *, W. Deng, G.P. Karwasz, A. Zecca \\ Dipartimento di Fisica, Istituto Nazionale per la Fisica della Materia, Universita' di Trento, 38050 Povo, Italy \\ Received 24 July 2001; received in revised form 19 February 2002
}

\begin{abstract}
Doppler-broadening measurements of the electron-positron annihilation line in twenty-seven single-element samples are presented. A coincidence technique has been used to suppress the background and to evidence the contribution of positron annihilation with core electrons. Systematic dependences on the atomic number of the target material are found in ratio curves obtained dividing the measured spectra by the spectrum of a reference material. The positron lifetime technique has been used to detect the presence of positron traps in all the samples. The change in the highmomentum part of the annihilation line due to positron trapping is illustrated. The measured data are in a good qualitative agreement with recent theoretical calculation and constitute the most complete measurement series, up to now, to establish a future data-base for positron annihilation spectroscopy.
\end{abstract}

(C) 2002 Elsevier Science B.V. All rights reserved.

Keywords: Positron annihilation; Doppler broadening; Electron momentum distribution

\section{Introduction}

Positron annihilation spectroscopy (PAS) is a well-established technique to detect open volume and negatively charged defects in solids [1]. Positrons, injected in a solid, lose their energy in a few picoseconds and, after a diffusion motion, annihilate mostly in two $511 \mathrm{keV}$ gamma quanta with an electron of the medium. Due to their positive

\footnotetext{
${ }^{*}$ Corresponding author. Tel.: +39-0461-881552; fax: +390461-881696.

E-mail address: brusa@science.unitn.it (R.S. Brusa).
}

charge, positrons are efficiently trapped by open volume defects.

The two most frequently used techniques to detect and to investigate the properties of open volume defects and their interaction with the medium are the lifetime and the Doppler broadening techniques. If fast positrons from a radioactive nuclide are used, the mean properties of the material under investigation are measured in a layer several tens of micron thick [2]. Otherwise, if a slow positron beam is utilized, a depth scanning from the surface to a few microns is possible [1].

In the Doppler broadening technique the 511 $\mathrm{keV}$ annihilation line, broadened by the Doppler 
shift due to the total momentum of the positronelectron annihilation pair, is directly recorded. In the measured spectrum, which is the onedimensional momentum distribution of the annihilating positron-electron pair, two main regions can be distinguished: a low-momentum region close to the peak position (typically $0<p_{\mathrm{L}}<$ $3.0 \times 10^{-3} m_{0} c$, where $p_{\mathrm{L}}$ is the longitudinal momentum) which reflects annihilations with valence electrons and a high-momentum region toward the tails of the spectrum (typically $6.2 \times 10^{-3} m_{0} c<$ $\left.p_{\mathrm{L}}<15 \times 10^{-3} m_{0} c\right)$ due to annihilation with highmomentum electrons. Usually two integral parameters $(S$ and $W)$ are used to probe the two regions of the spectrum [3]. With these two parameters it is possible to extract information on the type and concentration of the open volume defects [4]. On the other hand, the shape of the 511 $\mathrm{keV}$ annihilation line is different for each element. In particular the core electrons maintain their atomic characteristic also in a solid making it possible to use the signal from the positron-core electron annihilation for chemically selecting the atoms. A drawback is that the positron annihilation probability with electrons of high momentum is very low and the amplitude of the signal is comparable with the background. As a consequence, a background reduction coincidence technique with two detectors must be used to extract information from the tails $\left(p_{\mathrm{L}}\right.$ up to $\left.50 \times 10^{-3} m_{0} c\right)$ of the spectrum $[5,6]$ and this requires an increase of the measurement time for each spectrum. With a more detailed analysis of the Doppler spectrum one can get more and unique information about the defect site. The wave function of a trapped positron overlaps with the atoms surrounding the defect and these atoms can be identified by analyzing the highmomentum part of the annihilation spectrum.

The possibility to identify not only the type of open volume defect, but also the atoms that surround the defect, makes the positron probe even more powerful. Recently the promising problem of analyzing the positron annihilation with core electrons has been attacked both experimentally and theoretically [7-25]. The chemical selectivity of the positron probe can be used to detect impurity atoms associated to open volume defects. Refs. $[7,8,10,22,23,25]$ have demonstrated the capability of the technique to identify the detailed atomic structure of vacancy-impurity complexes in semiconductors.

In order to identify atoms that surround an open volume defect, we need to know accurately the core annihilation signal from these atoms and to describe it by theoretical models. Until now, measurements and calculation $[9,12,24]$ of positron-core-electron momentum were done on some pure elements but the picture is far from being complete. Discrepancies exist between different sets of measured and calculated data [9,24]. To evidence the small differences of positron annihilation with core electrons of different materials we shall present the experimental momentum spectra reduced to "ratio-curves" obtained dividing the measured curves by the curve of a reference element [9]. In this work we have measured positron annihilation spectra for 27 pure elements and we will outline regularities in the position and amplitude of peaks in the ratio curves. The pure elements have been characterized from a positronic point of view (i.e. absence or presence of open volume defects) with lifetime measurements. The lifetime of a positron in the solid is inversely proportional to the electron density probed by the positron itself. In open volume defects the electron density is reduced and the lifetime of a trapped positron increases in comparison with the lifetime of a free positron annihilating in the bulk of the material. Defects with different open volumes can be distinguished because trapped positrons show different lifetimes. The change of the ratio curves due to the presence of defects will be discussed, showing how the ratio curves are very sensitive to a small amount of positron traps. Our results will also be compared, when possible, with recent theoretical calculations [24] and measurements [9].

\section{Experimental}

Twenty-seven pure elements have been investigated; some of them were in single crystalline form. The list of these elements and some characteristics of the measured samples are reported in Table 1. The chemical purity of the samples is given in column two. 
Table 1

Atomic number $Z$, measured elements, purity of the elements, temperature used for the thermal treatments $\left(T_{\mathrm{a}}\right)$, average positron lifetimes $\tau_{\mathrm{a}}$

\begin{tabular}{|c|c|c|c|c|c|c|c|c|}
\hline$Z$ & Elements & Purity $(\%)$ & $T_{\mathrm{a}}{ }^{\circ} \mathrm{C}$ & $\tau_{\mathrm{a}}(\mathrm{ps})$ & $\tau_{1}(\mathrm{ps})$ & $\tau_{2}(\mathrm{ps})$ & $I_{2}(\%)$ & $\tau(\mathrm{ps})$ \\
\hline 4 & $\mathrm{Be}$ & - & 1000 & 156 & $138 \pm 1$ & $298 \pm 7$ & $12.2 \pm 1$ & $137 / 142 / 213$ \\
\hline 5 & $\mathrm{~B}$ & 99.5 & 1100 & 209 & - & - & - & $-1-1-$ \\
\hline 6 & C (graphite) & 99.5 & 1100 & 264 & $170 \pm 3$ & $343 \pm 3$ & $54.6 \pm 1$ & $-/ 215 /-$ \\
\hline 12 & $\mathrm{Mg}$ & - & 250 & 224 & - & - & - & $237 / 225 / 230$ \\
\hline 13 & $\mathrm{Al}\left(\begin{array}{lll}1 & 1 & 1\end{array}\right)$ & 99.9 & 500 & 160 & - & - & - & $166 / 163 / 166$ \\
\hline 14 & Si $p\left(\begin{array}{lll}1 & 0 & 0\end{array}\right)$ & - & - & 220 & - & - & - & $221 /-/ 228$ \\
\hline 22 & $\mathrm{Ti}$ & 99.9 & 1100 & 145 & - & - & - & $146 / 147 / 148$ \\
\hline 23 & $\mathrm{~V}$ & 99.7 & 1100 & 123 & - & - & - & $116 / 130 / 125$ \\
\hline 24 & $\mathrm{Cr}$ & 99.5 & 1100 & 157 & $83.1 \pm 1$ & $248 \pm 2$ & $44.8 \pm 1$ & $101 / 120 /-$ \\
\hline 25 & $\mathrm{Mn}$ & 99.5 & - & 150 & $121 \pm 1$ & $279 \pm 6$ & $17.7 \pm 1$ & $103 /-/-$ \\
\hline 26 & $\mathrm{Fe}$ & 99.99 & 1000 & 123 & $110.7 \pm 1$ & $257 \pm 10$ & $8.6 \pm 1$ & $101 / 106 / 107$ \\
\hline 27 & $\mathrm{Co}$ & 99.95 & 1100 & 117 & $99.6 \pm 1$ & $245 \pm 10$ & $11.7 \pm 1$ & $97 /-/ 119$ \\
\hline 28 & Ni ( $\left.\begin{array}{llll}1 & 1 & 1\end{array}\right)$ & 99.98 & 1000 & 105 & - & - & - & $96 / 110 / 110$ \\
\hline 29 & $\mathrm{Cu}\left(\begin{array}{lll}1 & 0 & 0\end{array}\right)$ & 99.8 & 850 & 115 & - & - & - & $106 / 110 / 122$ \\
\hline 30 & $\mathrm{Zn}\left(\begin{array}{llll}0 & 0 & 0 & 1\end{array}\right)$ & 99.9 & 250 & 149 & - & - & - & $134 / 148 / 160$ \\
\hline 31 & $\mathrm{Ga}$ & 99.9 & - & 237 & - & - & - & $-/ 190 / 194$ \\
\hline 32 & Ge $\left(\begin{array}{lll}1 & 0 & 0\end{array}\right)$ & - & - & 227 & - & - & - & $228 / 230 / 234$ \\
\hline 40 & $\mathrm{Zr}$ & 99.8 & 1100 & 159 & - & - & - & $159 / 165 / 165$ \\
\hline 41 & $\mathrm{Nb}$ & 99.8 & 1100 & 127 & - & - & - & $122 / 119 / 122$ \\
\hline 42 & Mo $\left(\begin{array}{lll}1 & 0 & 0\end{array}\right)$ & 99.9 & $>1500$ & 119 & - & - & - & $111 / 103 / 121$ \\
\hline 47 & $\mathrm{Ag}$ & 99.9 & 850 & 135 & - & - & - & $120 / 131 / 138$ \\
\hline 50 & $\mathrm{Sn}$ & 99.9985 & 150 & 197 & - & - & - & $275 / 289 / 201$ \\
\hline 72 & Hf & 99.5 & $>1500$ & 179 & - & - & - & $149 /-/-$ \\
\hline 74 & $\mathrm{~W}$ & 99.8 & $>2000$ & 115 & $103 \pm 1$ & $244 \pm 16$ & $8.6 \pm 1$ & $100 / 105 / 120$ \\
\hline 78 & $\mathrm{Pt}$ & 99.5 & 1000 & 167 & $142 \pm 1$ & $282 \pm 8$ & $18 \pm 1$ & $94 / 99 / 117$ \\
\hline 79 & $\mathrm{Au}$ & 99.99 & 850 & 123 & $119 \pm 2$ & $232 \pm 56$ & $3.7 \pm 1$ & $107 / 117 / 118$ \\
\hline 82 & $\mathrm{~Pb}$ & 99.5 & 250 & 206 & - & - & - & $187 / 194 / 214$ \\
\hline
\end{tabular}

The error on the average lifetimes is 1 ps. First $\left(\tau_{1}\right)$, second lifetime $\left(\tau_{2}\right)$ and the intensity $I_{2}$ of the second lifetime are reported for those elements in which the average lifetime was different from the bulk lifetime (columns from six to eight). In the last column collection of bulk lifetime $\tau$ from literature: first value (theoretical) from [30], second and third value (experimental) from [31,32] respectively.

The positron lifetime and the Dopplerbroadened $511 \mathrm{keV}$ annihilation line have been measured after annealing of the samples. In some cases measurements were also done on as-received samples. In general, the as-received samples can show presence of several types of positron traps: open volume defects from vacancies to vacancy clusters, dislocations, voids. All the samples were annealed for three hours in a vacuum of about $5 \times 10^{-7}$ mbar (annealing temperatures are given in third column of Table 1). Some of the samples $(\mathrm{Cr}, \mathrm{Co}, \mathrm{Au})$ have been annealed in two or more cycles of three hours. Mo, Hf, W were annealed at high temperatures in vacuum by electron bombardment. The samples were cooled down from the annealing temperature to room temperature in the oven by turning off the heating. Normally the entire cycle took more than $12 \mathrm{~h}$.

For lifetime and Doppler broadening measurements a ${ }^{22} \mathrm{Na}$ positron source was sandwiched between two equal samples. The ${ }^{22} \mathrm{Na}$ positron sources (0.37-0.6 MBq, prepared by deposition of ${ }^{22} \mathrm{NaCl}$ ) were sandwiched between two kapton foils $7 \mu \mathrm{m}$ thick.

The $511 \mathrm{keV}$ annihilation line is Dopplerbroadened $(511 \pm \Delta E)$ due to the longitudinal momentum $p_{\mathrm{L}}$ component of the annihilating positron-electron pair. In a Doppler experiment the longitudinal component $p_{\mathrm{L}}$ in the direction of the detector is measured. The momentum $p_{\mathrm{L}}$ is correlated to the Doppler shift $\Delta E$ by the formula $p_{\mathrm{L}}=2 \Delta E / c$ where $c$ is the light velocity. To study 
the annihilation with core electrons, the background must be reduced using a two-detector coincidence system [5]. In this experiment the main detector was a high purity Ge (HPGe) detector ( $16 \%$ efficiency) with a resolution of $1.3 \mathrm{keV}$ at 511 $\mathrm{keV}$ with $2 \mu$ s shaping time in the HPGe spectroscopy amplifier. A pileup rejection was used. The auxiliary detector supplying the coincidence signal was a $\mathrm{NaI}(\mathrm{Tl})$ scintillator. It was placed in collinear geometry with the Ge detector. The two detectors were shielded with lead in order to decrease the noise. The distance of the samples from the detectors was $23 \mathrm{~cm}$. With this set up, we get a coincidence count rate of about 140 counts/s in the 511 $\mathrm{keV}$ peak and a peak to background ratio of $1.5 \times 10^{4}$ on the high-energy side of the peak (see Fig. 1). The coincidence spectra were taken until more than $1 \times 10^{7}$ counts had been accumulated in the peak. We recall that using a $\mathrm{NaI}(\mathrm{Tl})$ detector coupled with the HPGe detector, only the background from the high-energy part of the $511 \mathrm{keV}$ line can be removed. The effects due to the incomplete charge collection, the Compton scattering and 3-gamma positronium decay cannot be eliminated in the low energy side of the peak. After each spectrum was recorded, the energy scale of the system was calibrated by taking the peak position

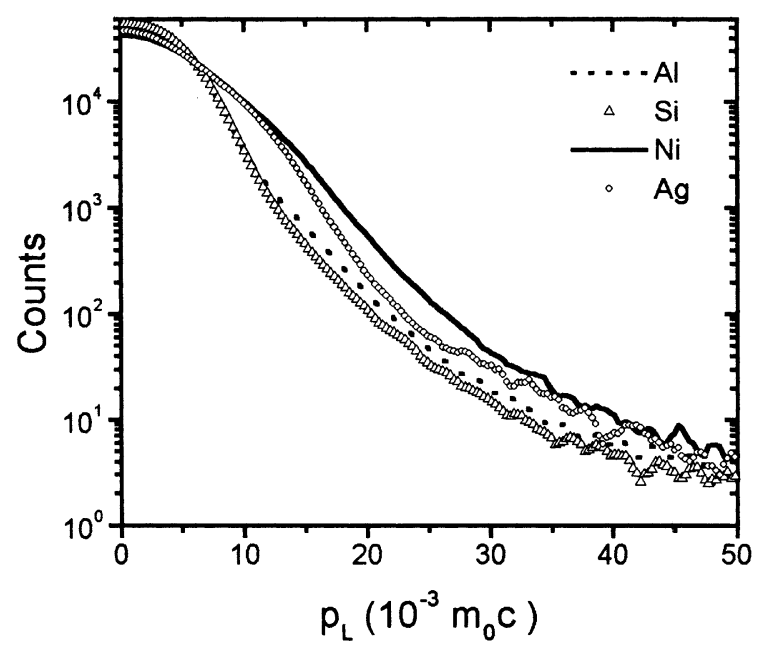

Fig. 1. Doppler spectra of $\mathrm{Al}, \mathrm{Si}, \mathrm{Ni}$ and $\mathrm{Ag}$ obtained by the coincidence technique. The curves, corrected for the background and the source component, are normalized to the same total area. of the $356.23 \mathrm{keV}$ gamma line supplied by a ${ }^{133} \mathrm{Ba}$ source in the single detector mode. In these spectra, each channel was $77.6 \mathrm{eV}$ wide.

Lifetime measurements have been done to check if the samples were defect free, as seen by positrons. The emission of a positron from a ${ }^{22} \mathrm{Na}$ radioactive source is followed by a $1.28 \mathrm{MeV}$ deexcitation photon. In a lifetime spectrometer this photon is used as a start signal and the $511 \mathrm{keV}$ annihilation photon as a stop signal. The positron lifetime measurements presented in this work, have been carried out with a fast-fast coincidence system [26] with Hamamatsu R2083Q photomultipliers and $\mathrm{BaF}_{2}$ scintillators. The resolution of the apparatus was 160 ps (FWHM) when measuring the positron lifetime. More than $1 \times 10^{6}$ counts have been accumulated in every lifetime spectrum. The positron lifetime is related to the delay between the positron emission and its annihilation. A lifetime spectrum is the sum of exponential decay components with different intensities. In a pure element without positron trapping centres, after correction for the source component, the lifetime spectrum is composed of a single exponential that gives the characteristic free positron annihilation lifetime in the bulk of the system $\tau$ [3]. In the following we have characterized our lifetime spectra by the average lifetime $\tau_{\mathrm{a}}$. Our $\tau_{\mathrm{a}}$ is evaluated with an error of about 1 ps. In the case of a pure element without defects, $\tau_{\mathrm{a}}$ equals $\tau$ (only one lifetime is present in the spectrum). If open volume defect are present, $\tau_{\mathrm{a}}$ is larger than $\tau$ of the element without defects.

\section{Analysis of the data}

First we consider positron lifetimes. The lifetime spectra have been analyzed using the Positronfit program [27]. To extract the positron lifetime in the sample, the fraction of positrons annihilating in the source and in the source support (source correction) must be subtracted from every spectrum. Kapton, here used as supporting material for the radioactive source, has a very simple lifetime spectrum with a single lifetime of 384 ps. In kapton no positronium is formed [28]. Because of the fraction of backscattered positrons from the 
samples changes with the atomic number $Z$ of the material, the source correction was done by subtracting the kapton lifetime with a different intensity according to the empirical formula given in $[3,29]$. The intensity of positron annihilating in the kapton foil spans from $4 \%$ for the lightest elements to $20 \%$ for the heaviest elements. A long lifetime component (1.5 ns) with a fixed intensity of $0.12 \%$, arising from positrons annihilating at the surfaces, was also subtracted from every spectrum. The average lifetime $\tau_{\mathrm{a}}$ measured after the last annealing are reported in column five of Table 1. By comparison, values from literature [30-32] are listed in column nine. To our knowledge the lifetime data in [30-32] are the only collection published until now. Note that all our lifetime data reported in Table 1 have been measured with the same set up and analyzed with the same procedure.

After repeated annealing some samples have shown residual positron trapping, their average lifetime are reported in bold characters in Table 1. For these samples we have also reported the two lifetimes $\left(\tau_{1}\right.$ and $\left.\tau_{2}\right)$, in which the spectrum was deconvoluted and the intensity $\left(I_{2}\right)$ of the second lifetime (columns from six to eight). For all these samples the second lifetime ranges from 240 to 300 ps and can be attributed to residual small open volume defects, most probably vacancy clusters formed for vacancy migration during heating [3].

The change in shape of the Doppler broadened spectrum due to annihilation with core electrons is small and, in many cases, it is difficult to appreciate directly the differences from one element to the other. As an example some selected spectra for pure elements are shown in Fig. 1. All the 27 measured curves approximately lie between the $\mathrm{Si}$ and the Ni curves.

Nevertheless, in order to enhance the differences among different spectra, it is common practice to construct ratio curves $(10,23,25)$. The spectra under examination are divided by the spectrum of a material chosen as the reference. The choice of the reference material determines the shape of the ratio curves: therefore the reference has to be chosen carefully. To give an example, investigating a series of iron alloys, a good choice could be to have a pure iron reference. In our case, willing to make a systematic work on many elements, the choice has been dictated by three criteria: first, the reference material has to be the same for all samples; second it has to be a low $Z$ element; third it has to be among those already used by previous authors - in order to allow the comparison with previous results. The use of a single low $Z$ reference element for all samples can make easier the comparison with theoretical results $(9,24)$. The spectra shown in Fig. 2 and following have been obtained as a ratio to the aluminum spectrum.

Starting with the element spectrum a constant background calculated as the average of the counts in 100 channels from 607 to $615 \mathrm{keV}$ was subtracted. Then a source correction was applied to every spectrum. We have prepared and measured two thick kapton samples. The Doppler spectrum of kapton was subtracted from all the measured spectra with a percentage weight dependent on atomic number of the sample and equal to the one used in the evaluation of the average lifetime reported in Table 1. Without this correction, errors up to $-15 \%$ in the height of the peaks of the ratio curves can be made in the heavier elements. Also the relative height of the peaks, where more than one peak is present, changes. Finally a smoothing routine has been applied (averaging over $\mathrm{n}$ channels, with $n$ increasing from 9 to 16 , going from 511 to $530 \mathrm{keV}$ ) to the corrected data and the spectra have been normalized to a total area of 1.0 from 511 to $530 \mathrm{keV}$ ( $p_{\mathrm{L}}$ from 0 to $74.3 \times 10^{-3} m_{0} c$ ). This smoothing procedure is necessary to extract the behaviour of the high-momentum tail of the spectrum, because the signal to noise on the counts in each channel decreases as the energy of the annihilation gamma increases (for a discussion see [10]). In our spectra an error of $3 \%$ is reached at about $516 \mathrm{keV}\left(p_{\mathrm{L}}\right.$ around $\left.23 \times 10^{-3} m_{0} c\right)$ and $12 \%$ at about $524 \mathrm{keV}\left(p_{\mathrm{L}}\right.$ around $\left.50 \times 10^{-3} m_{0} c\right)$. The corrected and normalized spectra have been finally divided by the reference spectrum.

In order to obtain a reference spectrum we have chosen two single crystal Al (1 111$)$ samples. As stressed in [21] it is important to record the orientation of the reference material because the ideal Doppler profile of $\mathrm{Al}$ contains a kink at the Fermi momentum that can affect the ratio curves. On the other hand, Doppler measurements reported on the same references on Al samples with two different 
orientation do not shown any appreciable difference. To minimize the noise on the ratio curves, particular care was taken to prepare the reference curve. First, a long measurement was performed accumulating about $3 \times 10^{7}$ counts. Then, after background subtraction and source correction, a nine points smoothing routine was applied and finally the long tail from $518 \mathrm{keV}$ was substituted by a two exponential fitting curve. This last step is justified by the fact that the Doppler broadening spectrum of $\mathrm{Al}$, as can be seen from theoretical calculation [7,24], decreases monotonically in the high-momentum region.

\section{Results and discussion}

\subsection{Pure elements}

The detailed shape of the high-momentum part of the Doppler spectra depends on the different contributions coming from the positron annihilation with the electrons of each shell of the atom. Positrons annihilate mainly with the electrons of the outermost shell due to the repulsion of the nucleus. Among the factors that determine the increase of the positron-electron annihilation probability at certain momenta we can remember: (i) the increase of the number of electrons at the individual shells; (ii) the localization in the $\mathbf{r}$ space of the electrons; more localized electrons gives a broad momentum distribution and vice versa; (iii) the crystalline structure. Calculations like in [7-9,24] will be needed for each element to set the partial contribution of positron-electron annihilation at the measured total momentum distribution and to the peaks appearing in the ratio curves. On the other hand phenomenological considerations can be done observing different groups of ratio curves and extrapolating the main results, obtained for some elements, in the above cited references.

We have grouped our Doppler broadening measurements on pure elements according to their position in the Periodic Table and to some similarities observed in their ratio-curve. The groups are the following: Be, B, C, Mg (Fig. 2), Ti, V, Cr, $\mathrm{Mn}, \mathrm{Fe}, \mathrm{Co}, \mathrm{Ni}, \mathrm{Cu}, \mathrm{Zn}$, Ga (Fig. 3(a) and (b)), Zr,
Nb, Mo, Ag (Fig. 4(a)), Hf, W, Pt, Au (Fig. 4(b)), Si, Ge, Sn, Pb (Fig. 5).

The Be, B, C curves in Fig. 2 show a single peak followed by a valley around $19.5 \times 10^{-3} m_{0} c$. For these light elements no theoretical calculations have been published. The peaks around $10 \times$ $10^{-3} m_{0} c$ of $\mathrm{Be}, \mathrm{B}$ and $\mathrm{C}$ can be correlated to the positron annihilation with the outermost electrons $2 p$. The peak rise with the number of $2 p$ electrons. $\mathrm{Mg}$ (with the 3s orbital filled) is the element in which positrons have the highest probability to annihilate with low-momentum electrons. This property reflects in a particular ratio curve with a maximum at zero momentum followed by a valley at $6 \times 10^{-3} m_{0} c$ and two peaks of decreasing intensity at $12.2 \times 10^{-3}$ and $20 \times 10^{-3} m_{0} c$.

In Fig. 3 (note the different scale for the vertical axis in Fig. 3(a) and (b)) the ratio curves for the transition metals of the fourth row in the periodic table are reported, together with the Ga data. The elements of this series are characterized by a welldefined peak slightly above $10 \times 10^{-3} m_{0} c$, with increasing intensity going from $\mathrm{Ti}$ to $\mathrm{Cu}$. Starting from vanadium an other shoulder manifests around $20 \times 10^{-3} m_{0} c$. This shoulder increases in amplitude and, going from $\mathrm{Fe}$ to $\mathrm{Cu}$ through $\mathrm{Co}$ and $\mathrm{Ni}$, broadens the ratio curves towards higher

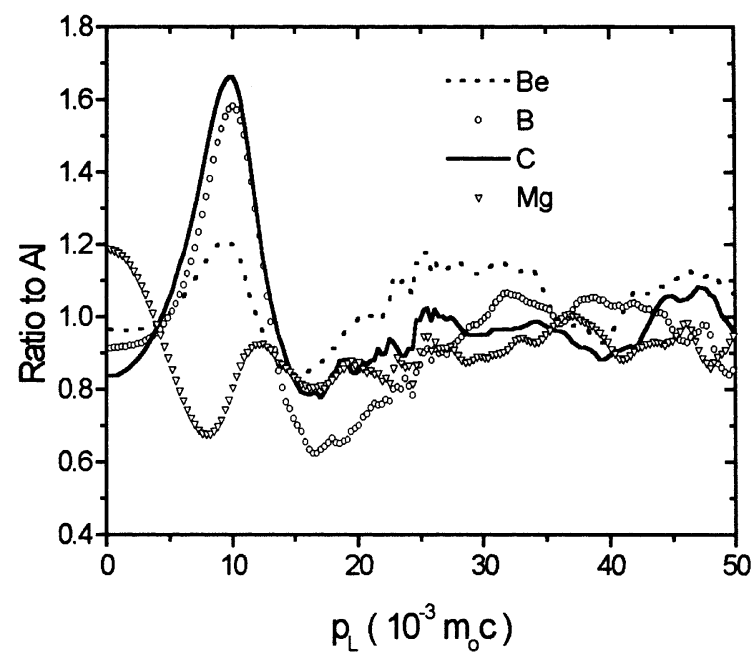

Fig. 2. Ratio of the experimental $\mathrm{Be}, \mathrm{B}, \mathrm{C}, \mathrm{Mg}$, positronelectron momentum distribution to the positron-electron momentum distribution of Al. 


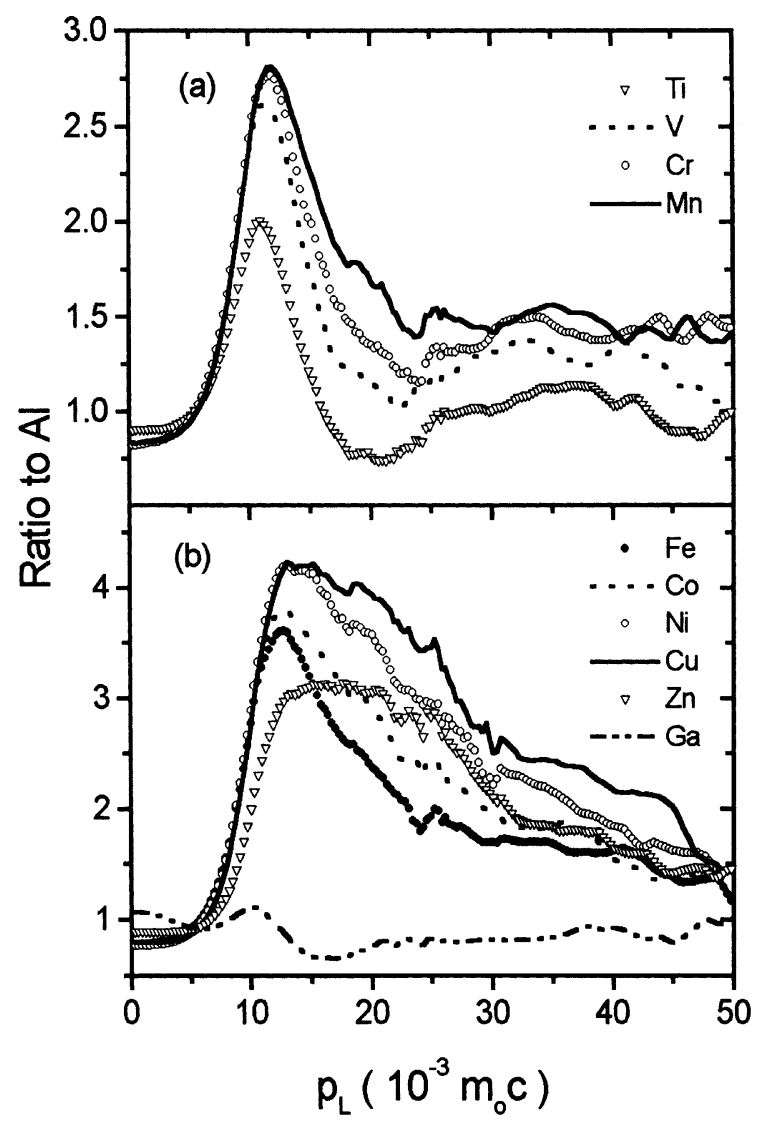

Fig. 3. Ratio of the experimental (a) Ti, V, Cr, Mn and (b) Fe $\mathrm{Co}, \mathrm{Ni}, \mathrm{Cu}, \mathrm{Zn}, \mathrm{Ga}$, positron-electron momentum distribution to the positron-electron momentum distribution of $\mathrm{Al}$.

momenta. The $\mathrm{Ni}$ and $\mathrm{Cu}$ ratio curves have the same value in the maximum and differentiate in the $\left(15<p_{\mathrm{L}}<30\right) \times 10^{-3} m_{0} c$ range where the $\mathrm{Cu}$ curve is higher. Also the peak position of the elements of this series moves progressively towards higher momenta. Calculations of positronelectron momentum distribution on $\mathrm{Cu}[8,24], \mathrm{Ni}$ and $\mathrm{Fe}$ [24] have shown that in the $\left(15<p_{\mathrm{L}}<\right.$ $40) \times 10^{-3} m_{0} c$ range the dominant contribution is due to positron annihilation with $3 \mathrm{~d}$ electrons. On the other hand, in $\mathrm{Cu}$ the $3 \mathrm{p}$ electrons give the dominant contribution above $40 \times 10^{-3} m_{0} c$. Extrapolating these results we can infer that the increase in the peak intensity $\left(10<p_{\mathrm{L}}<25 \times\right.$ $10^{-3} m_{0} c$ range) in the transition elements of Fig. 3 can be correlated with the increase of the annihilation of positrons with $3 \mathrm{~d}$ electrons: $\mathrm{Ti}, \mathrm{V}, \mathrm{Cr}$,

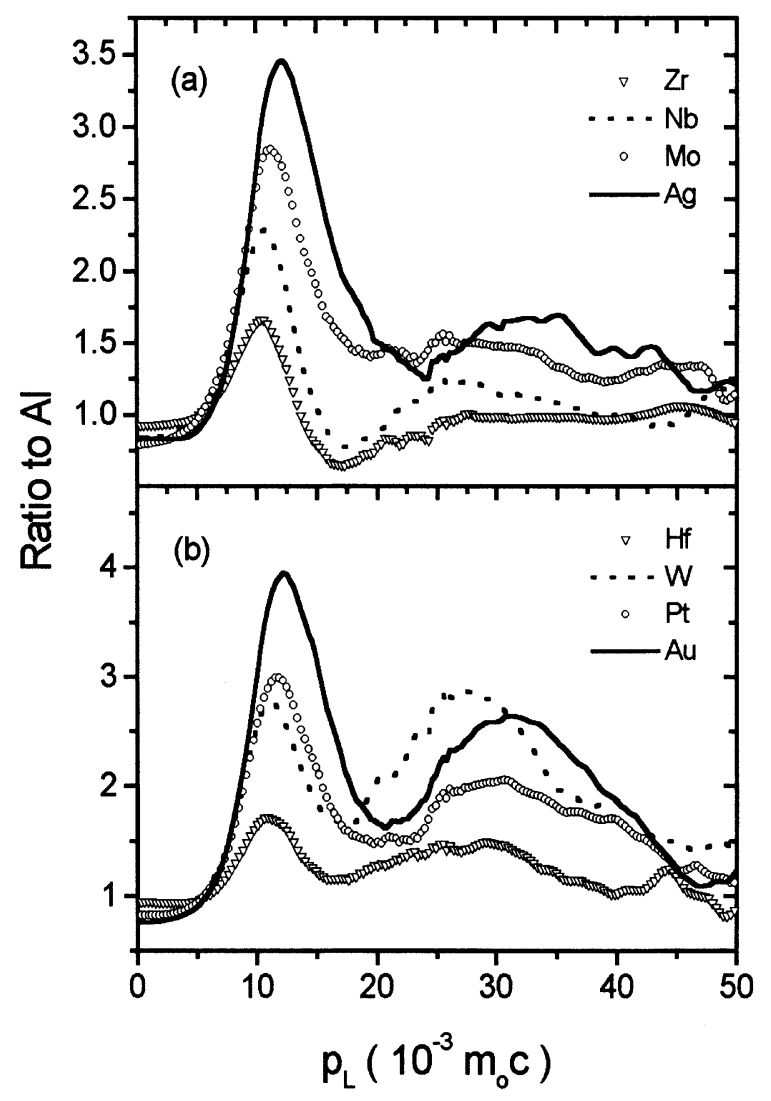

Fig. 4. Ratio of the experimental (a) $\mathrm{Zr}, \mathrm{Nb}, \mathrm{Mo}, \mathrm{Ag}$ and (b) $\mathrm{Hf}, \mathrm{W}, \mathrm{Pt}, \mathrm{Au}$, positron-electron momentum distribution to the positron-electron momentum distribution of $\mathrm{Al}$.

$\mathrm{Mn}, \mathrm{Fe}, \mathrm{Co}, \mathrm{Ni}, \mathrm{Cu}$ have 2, 3, 5, 5, 6, 7, 8, 10 electrons in the $3 \mathrm{~d}$ orbital respectively. The broadening of the peaks from $\mathrm{Fe}$ to $\mathrm{Cu}$ is probably due to the increasing contribution from the more localized $3 p$ electrons.

$\mathrm{Cr}, \mathrm{Mn}, \mathrm{Fe}, \mathrm{Co}$ samples used in this investigation have shown the presence of positron traps even after annealing (see Table 1). The relation among the height of the peaks is preserved even if we account for the presence of these traps. The $\mathrm{Zn}$ curve does not follow the trend observed in the ratio curves from $\mathrm{Ti}$ to $\mathrm{Cu}$ : it is very broad and lower than the Cu curve. This is possibly due to the complete filling of the $3 \mathrm{~d}$ and $4 \mathrm{~s}$ orbitals.

The $\mathrm{Ga}\left(29.7{ }^{\circ} \mathrm{C}\right.$ melting temperature $)$ ratio curve shows a high annihilation probability with valence electrons because of the presence of open 


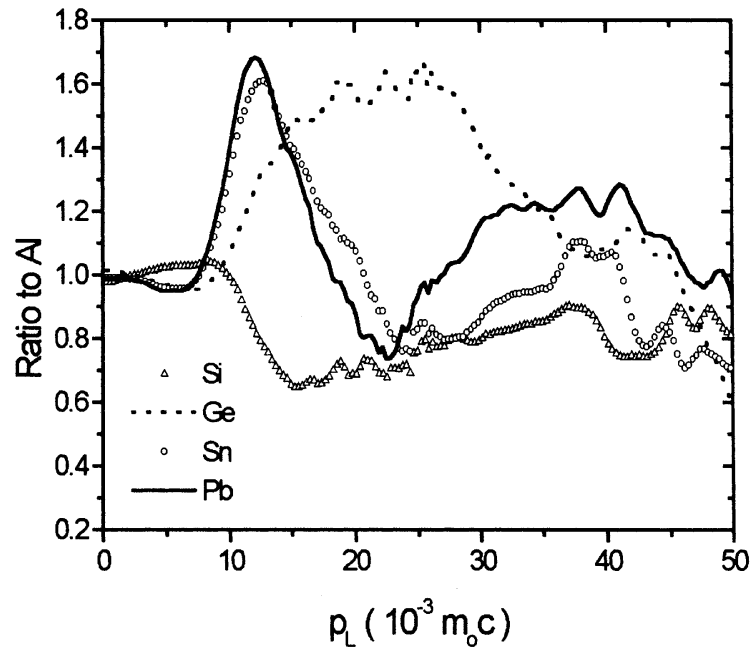

Fig. 5. Ratio of the experimental $\mathrm{Si}, \mathrm{Ge}, \mathrm{Sn}, \mathrm{Pb}$, positronelectron momentum distribution to the positron-electron momentum distribution of $\mathrm{Al}$.

volume defects in our samples (see the lifetime value in Table 1). This curve is characterized by a small peak at $10.2 \times 10^{-3} m_{0} c$.

Fig. 4(a) and (b) shows measurements on transition metals belonging to the fifth and sixth row of the Periodic Table. The ratio curves of these elements have similar features. Every ratio curve shows a first peak around $10-12 \times 10^{-3} m_{0} c$ followed by a second broad peak in the $(20<$ $\left.p_{\mathrm{L}}<40\right) \times 10^{-3} m_{0} c$ region. The position of the first peak moves, in both the series, from low to high momenta (Fig. 6). The second broad structure is more pronounced in the $\mathrm{Hf}-\mathrm{Au}$ series. The amplitudes of both peaks increase going from $\mathrm{Zr}$ to $\mathrm{Ag}$ and from $\mathrm{Hf}$ to $\mathrm{Au}$. The main contribution to the two peaks in the first series is due to the $4 \mathrm{~d}$ and $4 p$ electrons [24]: the increase in the second peak height going from $\mathrm{Zr}$ to $\mathrm{Ag}$ is probably due to the filling of the more delocalized $4 \mathrm{~d}$ orbital. In the case of the Hf-Au series the increase of the height of the two peaks can be correlated to the filling of the $5 \mathrm{~d}$ orbitals [24].

In Fig. 5 we have grouped $\mathrm{Si}$ and Ge semiconductors that have a diamond-like structure and $\mathrm{Sn}$ and $\mathrm{Pb}$ metals which show a similar shape in the ratio curves. In $\mathrm{Si}$ and $\mathrm{Ge}$ there is a large open volume between atoms due to the diamond-like

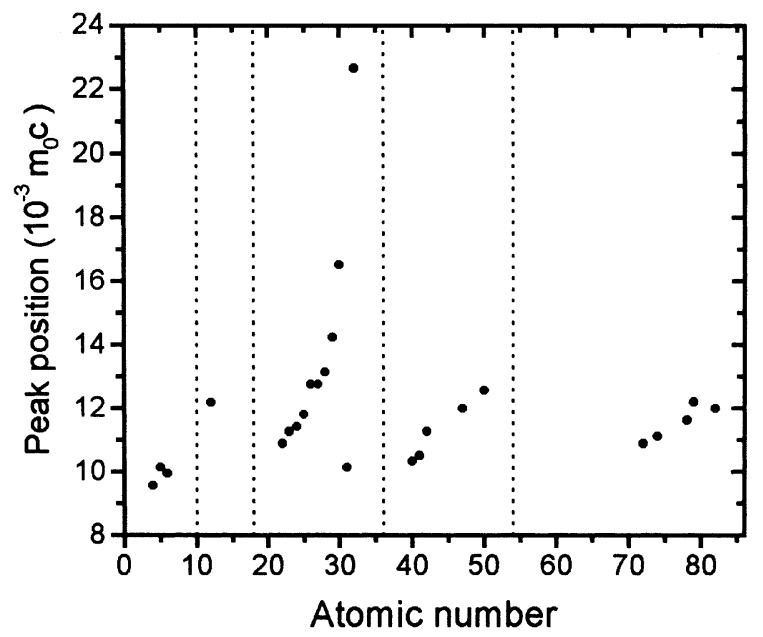

Fig. 6. Position of the first peak in the ratio between the positron-electron momentum distribution of the element to the positron-electron momentum distribution of $\mathrm{Al}$, versus the atomic number $Z$.

structure. In these elements the positron wave function is localized in this open structure and the probability of core annihilation is small. In Si the core annihilation is mainly due to $2 p$ electrons like in $\mathrm{Al}$, but the predicted annihilation rate is smaller than in $\mathrm{Al}$ [8]. For these reasons the $\mathrm{Si}$ ratio curve is almost flat up to $10 \times 10^{-3} m_{0} c$ and then drops below 1 in the region where the annihilation contribution from the $2 \mathrm{p}$ electrons is expected. In Ge the major contribution to core annihilation comes from positron annihilation with $3 \mathrm{~d}$ electrons well localized in the $\mathbf{r}$ space [8]. This manifests in the broad peak centered around $22 \times 10^{-3} m_{0} c$. The height of this peak is lower than the height of the peaks of transition metals in Fig. 3, which has been mainly attributed to annihilation with the $3 \mathrm{~d}$ electrons. In Sn (metallic $\alpha$ phase), the most important part of the core annihilation has been calculated to be due to $4 \mathrm{~d}$ electrons more localized in the momentum space [8]. This localization of the electrons in the momentum space lead to the more defined peak in Fig. 5. Extrapolating the results for $\mathrm{Sn}$ we expect that for $\mathrm{Pb}$, that shows a ratio curve very similar to that of $\mathrm{Sn}$, the annihilation comes mainly from $5 \mathrm{~d}$ electrons $(\mathrm{Sn}$ and $\mathrm{Pb}$ have the $4 \mathrm{~d}$ and $5 \mathrm{~d}$ orbitals filled and 4 valence electrons $5 \mathrm{~s}^{2} \mathrm{p}^{2}$ and $6 \mathrm{~s}^{2} \mathrm{p}^{2}$, respectively). We note 
that $\mathrm{Pb}$ and $\mathrm{Sn}$, like $\mathrm{Ga}$, have a low melting point, as a consequence the equilibrium concentration of thermal vacancies is expected at room temperature.

The data presented through Figs. 2-5 show as each element is characterized by features in the ratio curves: one or two peaks with different intensities and positions. The position of the first peak of all elements versus the atomic number $Z$ is summarized in Fig. 6. This plot evidences four groups, each corresponding to elements in the same row in the Periodic Table. In each group the peak position starts from low momenta and moves toward higher momenta. The height of the first peak (Figs. 2-5) follows the same behavior except for $\mathrm{Zn}, \mathrm{Ge}, \mathrm{Ga}, \mathrm{Sn}, \mathrm{Pb}$. The height and the position of the second peak, where detectable, seem to follow the same tendency as the first peak.

Although the height of the peaks has a discernible behaviour along the Periodic Table rows, its absolute value could be affected by positron annihilations in positron traps and this sets a limit to the present Doppler broadening measurements in pure elements. The height of the peak could be affected by: (a) positron annihilation with core electrons of impurity atoms (the purity of our samples is reported in Table 1) and/or annihilation in impurity-vacancy complexes; (b) positron annihilation into residual vacancies or vacancy agglomerates. The positron annihilations in such states, decreasing the annihilations with the core electrons of the element under study, reduce the intensity of the peaks in Figs. 2-5. This will be discussed in the following.

\subsection{Pure elements: effects due to positron trapping}

Annihilation of trapped positrons changes the probability of positron annihilation with core electrons and consequently the shape of the momentum spectrum. The high-momentum tail of the annihilation line is found to be very sensitive to a small perturbation caused by defects. In this paragraph we will present an experimental investigation of this effect on four samples.

In Fig. 7(a) and (b) the ratio curves for zirconium and niobium, before and after the thermal annealing are presented. One annealing only was

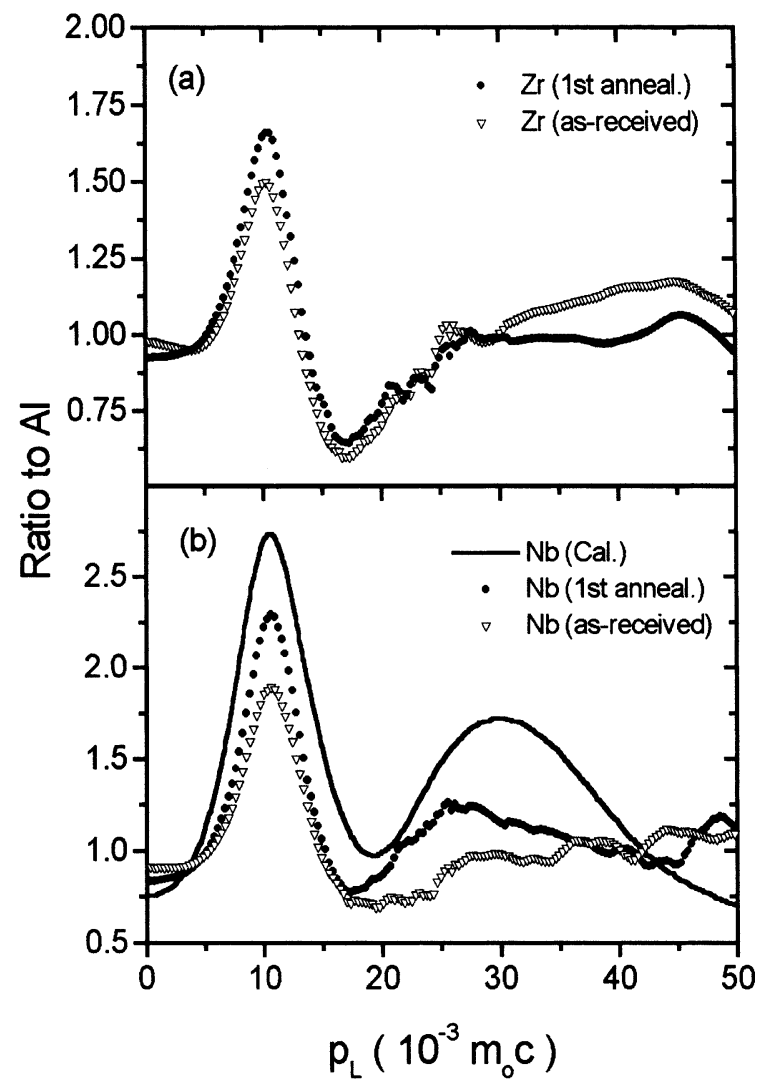

Fig. 7. Ratio of the experimental positron-electron momentum distribution to positron-electron momentum distribution of $\mathrm{Al}$ before and after annealing for (a) $\mathrm{Zr}$ (b) $\mathrm{Nb}$. The continuous line is the calculated ratio curve from [24].

sufficient to eliminate the open volume defects from the as-received samples. Before annealing the decomposition of the lifetime spectrum for $\mathrm{Zr}$ samples gave two positron lifetimes: $\tau_{1}=154.4 \pm 2$ ps $\left(I_{1}=89.2 \pm 3 \%\right)$ and $\tau_{2}=269 \pm 18$ ps $\left(I_{2}=\right.$ $10.8 \pm 3 \%$ ), average lifetime $\tau_{\mathrm{a}}=167 \mathrm{ps}$. After annealing, a single lifetime of 159 ps was found in very good agreement with the free defect bulk lifetime (see last column of Table 1). The ratio curve for the annealed samples increases in the 5$30 \times 10^{-3} m_{0} c$ region without changing the shape and this increase corresponds to the disappearance of small vacancy agglomerates.

In $\mathrm{Nb}$ before annealing only one lifetime was present (173 ps), indicating the presence of vacancy defects. After annealing this lifetime decreases to 
127 ps (free defect bulk lifetime). In the $\mathrm{Nb}$ ratio curves, the second peak, due mainly to annihilations with $4 \mathrm{~d}$ and $4 \mathrm{p}$ electrons [24], is partially suppressed by positron annihilation in trapping centers.

The ratio curves of $\mathrm{Zr}$ represent a typical behavior of the ratio curves in the majority of pure elements during the removing the open volume defects. An increase of the amplitude of the highmomentum part of the ratio spectra but without variation in the shape was also observed in other elements like $\mathrm{Ni}, \mathrm{Fe}, \mathrm{Cu}, \mathrm{Zn}, \mathrm{Mo}$. In these elements the presence of defects influences the total annihilation probability with core electrons.

As a second example in Fig. 8(a) and (b) we report the ratio curves for $\mathrm{W}$ and $\mathrm{Au}$. In $\mathrm{W}$ and

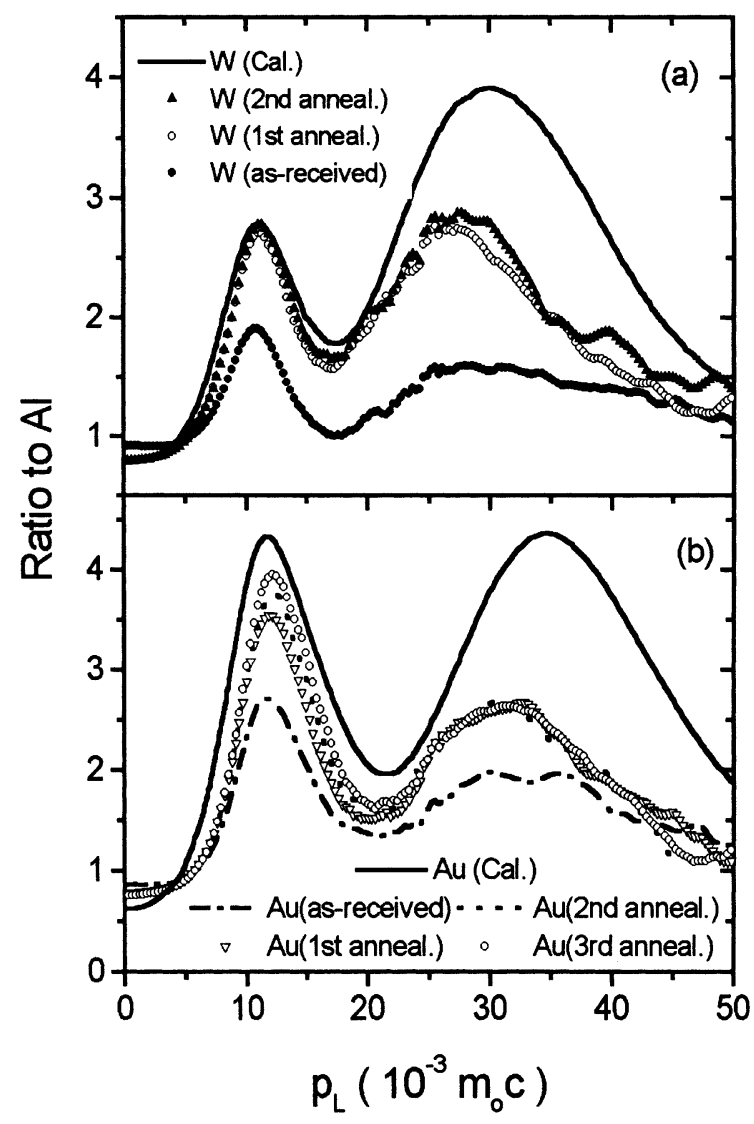

Fig. 8. Ratio of the experimental positron-electron momentum distribution to positron-electron momentum distribution of $\mathrm{Al}$ before and after annealing for (a) W (b) Au. The continuous lines are calculated ratio curves from [24].
Au more than one annealing was done but this was not sufficient to eliminate all the positron traps in our samples. Despite of this, the ratio curves for these elements are well suited to show different characteristic trends during the annealing procedure and are representative of the behavior of the ratio curves of other elements.

The lifetime spectra in the $\mathrm{W}$ as received samples, consist of a single lifetime of 175 ps, indicating that our starting sample is rich of small open volume defects [32]. The first annealing causes a big effect: the intensity of the two peaks increases. The second annealing produces a further small increase in the intensity of the peaks. In particular the second peak slightly surpasses in height the first peak. The lifetime spectra measured after the two annealing have been decomposed in two components. The values obtained for the lifetimes and their intensities were, within the errors, the same after the first and the second annealing (see Table 1 for the values). On the contrary, as can be seen from Fig. 8(a), the two ratio curves differ. In this case the ratio curves are more sensitive than the lifetime analysis to a small change in the defect concentration and/or defect type.

The first annealing in $\mathrm{Au}$ produces a big increase in the intensity of the two peaks. The starting material was rich of several positron traps $\left(\tau_{1}=181 \pm 3, \quad I_{1}=92 \pm 5, \quad \tau_{2}=291 \pm 37\right.$, $I_{2}=8 \pm 5$ ). After the first annealing an increase of the amplitude of the two peaks is observed. Differently than in W case, the second and third annealing have major influence on the first peak, with no influence on the second one. After the first annealing $\tau_{1}$ decreases to $118 \pm 1$ ps $\left(I_{1}=93 \pm 1\right)$ and $\tau_{2}$ to $256 \pm 16 \mathrm{ps}\left(I_{2}=7 \pm 1\right)$. Like in the $\mathrm{W}$ case, the second annealing has not produced a variation in the lifetime spectra, but we observe in Fig. 8(b) a big increase of the intensity of the first peak. The last annealing (see lifetime data in Table 1) is characterized by a change in the intensity of the two lifetime component. In the $\mathrm{Au}$ case it is evident the movement of the position of the first peak towards higher momenta, during the removal of open volume defects. This behavior in the peak position was found in other elements like $\mathrm{Cr}$ and $\mathrm{Co}$. 


\subsection{Comparisons with other results}

In Ref. [24], theoretical ratio-curves to $\mathrm{Al}$ were reported for $\mathrm{Fe}, \mathrm{Ni}, \mathrm{Cu}, \mathrm{Zn}, \mathrm{Ge}, \mathrm{Nb}, \mathrm{Mo}, \mathrm{Ag}, \mathrm{Sn}$, $\mathrm{W}, \mathrm{Au}, \mathrm{Pb}$. Experimental ratio curves to $\mathrm{Al}$ were reported for $\mathrm{Cu}, \mathrm{Ni}, \mathrm{Fe}, \mathrm{W}, \mathrm{Ge}, \mathrm{C}, \mathrm{Sn}, \mathrm{Si}$ [9]. Our experimental results agree qualitatively both with the theoretical and the measured curves. But, before outlining the major similarities and differences, some points must be stressed. It has been shown in a case study on $\mathrm{Cu}$ [21] that the ratio curves change if HPGe detectors of different energy resolutions are used to acquire the momentum spectra. The general shape of the ratio curve remains the same by worsening the detector resolution but the ratio curve decreases in intensity and moves towards higher momenta. We have measured our samples with a detector resolution of $1.3 \mathrm{keV}$, consequently the peaks in our ratio curves can be expected to be slightly higher than the peaks in the ratio curves calculated convoluting the theoretical results with a gaussian with $1.5 \mathrm{keV}$ FWHM to simulate the detector resolution [24] and higher than the measurements obtained with such detector resolution [9]. The model used to make the theoretical calculation reported in [24], as outlined by the authors, does not give a good description of the valence electrons and the calculated ratio curves can be only used for qualitative comparison below $20 \times 10^{-3} m_{0} c$.

The theoretical ratio curves of [24] are higher than our experimental data but the general shape, the displacement of the first peak and the increase of its amplitude with the position of the elements in the periodic table are in a good agreement with our findings. For a comparison with our experimental data we have reported theoretical curves, from [24], for elements belonging to different rows in the periodic table. $\mathrm{Ni}$ and $\mathrm{Pb}$ ratio curves are shown in Fig. 9, Nb curve in Fig. 7 and $\mathrm{W}$ and $\mathrm{Au}$ curves in Fig. 8. Above $20 \times$ $10^{-3} m_{0} c$ the shape of all our measured curves is in agreement with theory. The maximum of the second peak in $\mathrm{Nb}, \mathrm{W}$ and $\mathrm{Au}$ is shifted toward lower momenta as expected from considerations on the detector resolution. The theoretical model, as said, is not precise below $p_{\mathrm{L}}=20 \times 10^{-3} m_{0} c$, but we stress the similarity between theoretical

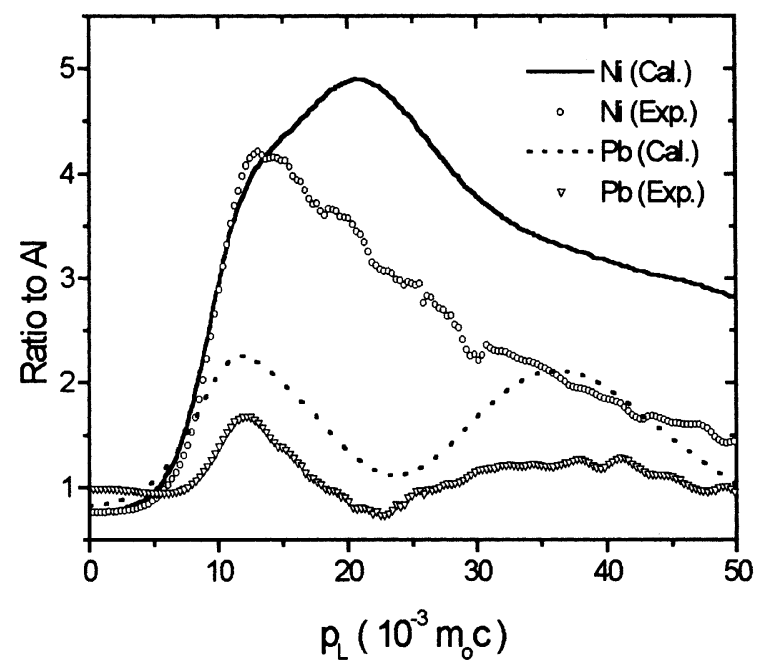

Fig. 9. Comparisons between theoretical (from [24]) and present experimental positron-electron momentum distribution ratio-curves with $\mathrm{Al}$, for $\mathrm{Ni}$ and $\mathrm{Pb}$.

and experimental curves also in this momentum region.

The overall agreement is found also with the measurements of [9]. Here, the position of the maxima are slightly displaced towards higher momenta as expected by the different detector resolution [21]. The peaks are generally higher than ours. The change in the height of the peaks is in the opposite direction than expected from considerations on detector resolution only. Similar differences were stressed between results obtained in $[8,9]$ and can be attributed to the differences in the experimental set-ups: in [9] a two Ge detector system was used; in [8] a $\mathrm{NaI}(\mathrm{Tl})$ combined with a Ge detector was used as in the present measurement. Part of the difference in the height of the ratio curves, especially at high momenta $\left(p_{\mathrm{L}}>20 \times 10^{-3} m_{0} c\right)$, can be ascribed to a higher background in the NaI(Tl)-Ge system and probably to a different content of residual positron traps. Unfortunately in the previous papers published on this subject the purity of the measured samples is reported only in few cases. More reliable calculations below $20 \times 10^{-3} m_{0} c$ and measurements on samples of different purity, will be useful for more accurate comparisons. We finally note that the FWHM of the peaks in the ratio curves is smaller 
with a better detector resolution, which is the case of the present measurements.

\section{Conclusions}

Recent works $[22,23,25]$ have shown that impurity elements give a precise signature in the Doppler broadened $511 \mathrm{keV}$ annihilation line when positrons annihilate in impurity-defect complexes. This signature can be evidenced in momentum spectra measured by a two-detector coincidence technique. Here the position of the peaks is characteristic of the element impurities surrounding the annihilation site.

This paper provides the first large collection of measurements which can be used in the identification of elements and that constitute the starting point for constituting a future database. Elemental samples used in the measurement were of good purity and have been characterized by positron lifetime measurements. This work shows that the two-detector coincidence technique is very sensitive to the presence of positron traps in the samples and that it is more sensitive than the lifetime technique in the detection of positron traps. This finding has to be taken into account for precise comparisons among different sets of experimental data or between theory and experiment.

\section{Acknowledgements}

One of us (W.D.) thanks INFM for the two years grant. The work was partially supported by the Ministero della Università e della Ricerca Tecnologica, Cofin 2000 program MM02178957002 .

\section{References}

[1] A. Dupasquier, A.P. Mills Jr. (Eds.), Positron Spectroscopy of Solids, North Holland, Amsterdam, 1995.

[2] W. Brandt, A. Dupasquier (Eds.), Positron Solid-State Physics, North Holland, Amsterdam, 1983.

[3] P. Hautojärvi, C. Corbel, in: A. Dupasquier, A.P. Mills Jr. (Eds.), Positron Spectroscopy of Solids, North Holland, Amsterdam, 1995, p. 491.
[4] R.S. Brusa, G.P. Karwasz, N. Tiengo, A. Zecca, F. Corni, R. Tonini, G. Ottaviani, Phys. Rev. B 61 (2000) 10154.

[5] K.G. Lynn, A.N. Goland, Solid State Commun. 18 (1976) 1549.

[6] K.G. Lynn, J.R. MacDonald, R.A. Boie, L.C. Feldman, J.D. Gabbe, M.F. Robbins, E. Bonderup, J. Golovchenko, Phys. Rev. Lett. 38 (1977) 241.

[7] M. Alatalo, H. Kauppinen, K. Saarinen, M.J. Puska, J. Mäkinen, P. Hautojärvi, R.M. Nieminen, Phys. Rev. B 51 (1995) 4176.

[8] M. Alatalo, B. Barbiellini, M. Hakala, H. Kauppinen, T. Korhonen, M.J. Puska, K. Saarinen, P. Hautojärvi, R.M. Nieminen, Phys. Rev. B 54 (1996) 2397.

[9] P. Asoka-Kumar, M. Alatalo, V.J. Ghosh, A.C. Kruseman, B. Nielsen, K.G. Lynn, Phys. Rev. Lett. 77 (1996) 2097.

[10] S. Szpala, P. Asoka-Kumar, B. Nielsen, J.P. Peng, S. Hayakawa, K.G. Lynn, H.-J. Gossmann, Phys. Rev. B 54 (1996) 4722.

[11] U. Myler, R.D. Goldberg, A.P. Knights, D.W. Lawther, P.J. Simpson, Appl. Phys. Lett. 69 (1996) 3333.

[12] U. Myler, P.J. Simpson, Phys. Rev. B 56 (1997) 14303.

[13] H. Kauppinen, L. Baroux, K. Saarinen, C. Corbel, P. Hautojärvi, J. Phys. Condens. Matter 9 (1997) 5495.

[14] K. Saarinen, H. Kauppinen, T. Laine, P. Hautojärvi, Appl. Surf. Sci. 116 (1997) 273.

[15] V.J. Ghosh, M. Alatalo, P. Asoka-Kumar, K.G. Lynn, A.C. Kruseman, Appl. Surf. Sci. 116 (1997) 279.

[16] B. Barbiellini, M.J. Puska, M. Alatalo, M. Hakala, A. Harju, T. Korhonen, S. Siljamaki, T. Torsti, R.M. Nieminen, Appl. Surf. Sci. 116 (1997) 283.

[17] A.C. Kruseman, H. Schut, A. van Veen, P.E. Mijanarends, M. Clement, J.M.M. de Nijs, Appl. Surf. Sci. 116 (1997) 192.

[18] P.E. Mijnarends, A.C. Kruseman, A. van Veen, V.J. Ghosh, P. Asoka-Kumar, A. Basil, S. Kaprzyk, K.G. Lynn, Mater. Sci. Forum. 255-257 (1997) 784.

[19] C. Kruseman, H. Schut, M. Fujinami, A. van Veen, Mater. Sci. Forum. 255-257 (1997) 793.

[20] M. Alatalo, P. Asoka-Kumar, V.J. Ghosh, B. Nielsen, K.G. Lynn, A.C. Kruseman, A. van Veen, T. Korhonen, M.J. Puska, J. Phys. Chem. Solids 59 (1998) 55.

[21] V.J. Ghosh, B. Nielsen, A.C. Kruseman, P.E. Mijanarends, A. van Veen, K.J. Lynn, Appl. Surf. Sci. 149 (1999) 234.

[22] K. Saarinen, J. Nissilä, H. Kauppinen, M. Hakala, M.J. Puska, P. Hautojärvi, C. Corbel, Phys. Rev. Lett. 82 (1999) 1883.

[23] M.P. Petkov, M.H. Weber, K.G. Lynn, R.S. Crandall, V.J. Ghosh, Phys. Rev. Lett. 82 (1999) 3819.

[24] V.J. Ghosh, M. Alatalo, P. Asoka-Kumar, B. Nielsen, K.G. Lynn, A.C. Kruseman, P.E. Mijnarends, Phys. Rev. B 61 (2000) 10092.

[25] R.S. Brusa, W. Deng, G.P. Karwasz, A. Zecca, D. Pliszka, Appl. Phys. Lett. 79 (2001) 1492.

[26] J. De Vries, A. Zecca, R.S. Brusa, R.G. Grisenti, S. Oss, Nucl. Instr. and Meth. A 275 (1989) 194.

[27] P. Kirkegaard, N.J. Pedersen, M. Eldrup, PATFIT-88, Riso-M-2740 (1989). 
[28] R.S. Brusa, A. Dupasquier, E. Galvanetto, A. Zecca, Appl. Phys. A 54 (1992) 233.

[29] M. Bertolaccini, L. Zappa, Nuovo Cimento B 52 (1967) 487.

[30] M.J. Puska, R. Nieminen, Rev. Mod. Phys. 66 (1994) 841.
[31] A. Seeger, F. Barnhart, W. Bauer, in: L. DorikensVanpraet, M. Dorikens, D. Segers (Eds.), Positron Annihilation, World Scientific, Singapore, 1989, p. 275.

[32] I.K. MacKenzie, in: W. Brandt, A. Dupasquier (Eds.), Positron Solid-State Physics, North Holland, Amsterdam, 1995, p. 196. 